\title{
CORRECTIONS
}

\section{Publisher Correction: Language, numeracy and logic in microbiome science}

\section{Fergus Shanahan (iD) and Colin Hill}

Nature Reviews Gastroenterology \& Hepatology (2019)

https://doi.org/10.1038/s41575-019-0163-5 Published online 05 June 2019

In the original version of this article published online the affiliation for Colin Hill was

incorrectly listed. This error has been corrected in the HTML and PDF of the manuscript.

https://doi.org/10.1038/s41575-019-0170-6 I Published online 12 June 2019 